the system, emergency call workers asked ambulance callers for the patient's age, chief complaint, speaking ability and previous history of illness. We analysed the relation between this information and the disease classification (ICD-10) coded by physicians in the emergency department at each patient's destination. A logistic model was used to estimate the probability of stroke occurrence at the time of emergency calls.

Results There were 66744 patients who were transported by ambulance during the study period. Among the 66744 cases, this study targeted 60791 patients whose triage data were complete. Out of these 60791 cases, 2496 were coded as suffering a stroke (ICD-10: I60-I69) by emergency department physicians. The probability of stroke occurrence from the information given at the time of the emergency call was estimated quantitatively.

Conclusion Stroke occurrence can be expressed as a probability (\%) at the time of the emergency call. The probability can be used to establish a quick and smooth transport of patients to appropriate medical facilities.

\section{SP3-24 SOCIOECONOMIC DETERMINANTS OF SPORADIC CAMPYLOBACTER JEJUNI INFECTIONS AMONG CHILDREN IN GREECE}

doi:10.1136/jech.2011.142976o.24

\begin{abstract}
${ }^{1} \mathrm{~K}$ Mellou, ${ }^{*} 1,2 \mathrm{G}$ Saroglou, ${ }^{2} \mathrm{E}$ Velonakis. ${ }^{1}$ Hellenic Centre for Diseases Control and Prevention, Athens, Greece; ${ }^{2}$ National and Kapodistrian University of Athens, Athens, Greece
\end{abstract}

Introduction Campylobacter spp. is a common cause of acute bacterial gastroenteritis worldwide but the source of the infection in many human sporadic cases remains unknown. Many case-control studies have been conducted, however, little has been published about socioeconomic differences in the incidence of Campylobacter infection.

Methods From December 2004 to December 2006 a prospective matched case-control study was conducted, in the urban area of Attica, Greece, in order to identify risk factors for sporadic Campylobacter jejuni infections in children aged $<15$ years.

Results A total of 205 bacteriologically confirmed cases and 205 controls matched by age group ( $<1$ year, 1 to 4,5 to 9 and 10 to 14 years) and gender were included in the study. After univariate analysis two conditional logistic regression multivariate models were fitted. The first included "protective" variables, whereas these were ignored in the second. Apart from the consumption of chicken the week prior to disease onset, some socioeconomic risk factors of the disease were also recognised. Ethnicity, mother's employment status and, living in a house with a garden were found to be independently associated with the disease occurrence in both models. Domestic travel was correlated to the frequency of the disease only in model II.

Conclusion Apart from the dietary determinants of Campylobacter infection that have been studied thoroughly in a number of studies, there are specific socioeconomic characteristics that could either be risk factors of the disease or just hide other behavioural and eating risk factors.

\section{SP3-25 PAROXYSMAL ATRIAL FIBRILLATION PICTURED BY IMPLANTABLE DEVICES WITH REMOTE MONITORING}

doi:10.1136/jech.2011.1429760.25

N Varma. ${ }^{*}$ Cleveland Clinic, Cleveland, Ohio, USA

Background Atrial fibrillation (AF) is an epidemic disease. Outcomes and treatment are determined by symptoms and risk posed.
These may be influenced by absolute AF burden, degree of temporal dispersion of episodes, and/or progression to persistent arrhythmia. Characterisation is challenging with traditional intermittent ECG monitoring because the arrhythmia is evanescent and largely asymptomatic. Implanted devices with remote monitoring (RM) mechanisms may enable automatic continuous monitoring with accurate arrhythmia resolution. This was tested.

Methods and Results 276 consecutive patients with RM pacemakers were followed for $12 \pm 2$ months, 29 experienced at least 1 day with AF. In each patient, AF days were classed by mode switch duration (irrespective of mode switch number) according to $>6,>12,>18$ and $>24 \mathrm{~h} /$ day. AF burden differed among and within individuals for example, one patient had only one AF day contrasting with 93 in another. In patient 15, 81/93 days were associated with $>18 \mathrm{~h}$ of AF that is, AF burden was heavy when it occurred, but patient 20 had 7 AF days but none exceeded $18 \mathrm{~h}$ duration per day. Similar variabilities were observed in heart failure patients (TRUST trial). Though conventionally considered at high risk for AF, $<10 \%$ patients developed AF of $>2 \mathrm{~h} /$ day in 1 year.

Conclusion Wide diversity in AF arrhythmia load likely impart different risks and require different treatment. Understanding may be enhanced by continuous remote monitoring and permit reevaluation of current clinical management guidelines.

\section{SP3-26 PREVALENCE AND CONSULTATION RATES OF LIFE-STYLE RELATED DISEASES IN JAPANESE MIDDLE-AGED AND ELDERLY WOMEN}

doi:10.1136/jech.2011.142976o.26

${ }^{1,2} \mathrm{~F}$ Ando, ${ }^{2} \mathrm{M}$ Takemura, ${ }^{2} \mathrm{Y}$ Matsui, ${ }^{2} \mathrm{H}$ Shimokata. ${ }^{1}$ Aichi-Shukutoku University, Nagakute, Japan; ${ }^{2}$ National Center of Geriatrics and Gerontology, Obu, Japan

Introduction Prevalence rates (PR) and consultation rates (CR) of life-style related diseases are very important for public health but studies for them in women are far less than those in men in Japan.

Purpose To estimate the PR and CR of life-style related diseases in Japanese middle-aged and elderly women.

Methods The subjects were 1194 women aged 40 to 87, randomly selected from a resident registration. Japanese diagnosis criteria for diseases were used to determine the PR. The CR was defined as the ratio of the number of subjects consulting medical doctor regularly to the whole subjects. The PR, CR and the ratio of CR to PR (CR) $\mathrm{PR}$ ) of diseases were calculated in the whole subjects and by agedecade groups.

Results The PR of anaemia, diabetes, dyslipidemia, hypertension, osteoporosis, urinary incontinence, obesity and emaciation were $0.105,0.082,0.556,0.328,0.208,0.341,0.195$ and 0.075 , while CR of the first six diseases were $0.016,0.053,0.140,0.263,0.079$ and 0.009 , respectively. The CR/PR distributed widely from 0.026 (urinary incontinence) to 0.803 (hypertension). The PR of dyslipidemia, diabetes and obesity were the highest in the 60s and 70s, whereas those of hypertension and osteoporosis increased exponentially with ageing. The PR of anaemia showed U-shape but the $\mathrm{CR} / \mathrm{PR}$ decreased with ageing. The PR of urinary incontinence were over 0.30 in all age-decade groups but no one consulted medical doctors in the 40s and 50s.

Conclusion The PR of diseases and the attitude to medication change with ageing in Japanese middle-aged and elderly women 